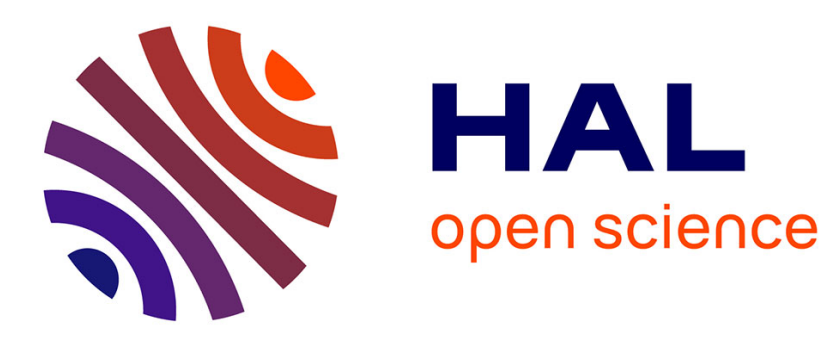

\title{
Direct comparison between in situ and radar measurements of temperature fluctuation spectra: A puzzling result
}

Francis Dalaudier, Michel Crochet, Claude Sidi

\section{To cite this version:}

Francis Dalaudier, Michel Crochet, Claude Sidi. Direct comparison between in situ and radar measurements of temperature fluctuation spectra: A puzzling result. Radio Science, 1989, 24 (3), pp.311-324. 10.1029/RS024i003p00311 . insu-03580842

\section{HAL Id: insu-03580842 \\ https://hal-insu.archives-ouvertes.fr/insu-03580842}

Submitted on 18 Feb 2022

HAL is a multi-disciplinary open access archive for the deposit and dissemination of scientific research documents, whether they are published or not. The documents may come from teaching and research institutions in France or abroad, or from public or private research centers.
L'archive ouverte pluridisciplinaire HAL, est destinée au dépôt et à la diffusion de documents scientifiques de niveau recherche, publiés ou non, émanant des établissements d'enseignement et de recherche français ou étrangers, des laboratoires publics ou privés.

$$
\text { Copyright }
$$




\section{Direct comparison between in situ and radar measurements of temperature fluctuation spectra: A puzzling result}

Francis Dalaudier

Service d'Aéronomie du Centre National de la Recherche Scientifique, Verrières le Buisson, France

Michel Crochet

Laboratoire de Sondages Electromagnétiques de l'Environnement Terrestre, Université de Toulon, France

Claude Sidi

Service d'Aéronomie du Centre National de la Recherche Scientifique, Verrières le Buisson, France

(Received June 20, 1988; revised December 19, 1988; accepted December 22, 1988.)

Investigations of temperature fluctuation spectra of atmospheric turbulence were performed simultaneously by remote sensing and in situ measurements. A balloon borne instrumented gondola measured temperature and velocity fluctuations up to an altitude of $18 \mathrm{~km}$. Spectral analysis of these data led to one-dimensional spectra. Simultaneously, the nearby stratospheretroposphere radar "Provence" obtained vertical profiles of reflectivity for both vertical and oblique $\left(15^{\circ}\right)$ lines of sight and for various range resolutions. According to the classical interpretation, the radar echoes result from the three-dimensional spectrum of refractive index fluctuations, and its aspect sensitivity reflects the spectrum anisotropy at the half radar wavelength. We thus separately reconstructed the reflectivity profiles for the two lines of sight. For the oblique one, the in situ observed spectral level of temperature fluctuations (assumed isotropic) was converted into radar reflectivity. For the vertical measurements, a theoretical "universal" level was used, along with an isotropic conversion formula (a tentative account for the known anisotropy is given in the appendix). Comparison of the reconstructed reflectivity profiles with the observed one proves to be very puzzling. For oblique measurements, where the reconstruction process uses only widely accepted hypotheses, the observed profiles are poorly reproduced. By contrast, for vertical measurements, the proposed model leads to a good reconstruction of the shape of the reflectivity profiles. In both cases, the predicted level is always strongly overestimated. The discussion concludes that the discrepancy is real and calls for further studies of this problem.

\section{INTRODUCTION}

The origin of the radar echoes in clear air conditions is known to be the small-scale inhomogeneities of the refractive index of air. Such inhomogeneities result mainly from humidity fluctuations in the lower troposphere and from temperature fluctuations in the higher troposphere and lower stratosphere. Echoes are also obtained in the mesosphere, as a result of inhomogeneities in the electronic density [Woodman and Guillen, 1974]. The stratosphere troposphere (ST) radars use theses echoes in order to study the lower atmosphere up to $\approx 20 \mathrm{~km}$.

If the determination of the radial velocity of the scattering medium is quite easy, the study of its turbulent state is still

Copyright 1989 by the American Geophysical Union.

Paper number 88RS04325.

0048-6604/89/88RS-04325\$08.00 under question. The theory of electromagnetic wave propagation in a turbulent medium [Tatarski, 1961] relates the backscattered power to the three-dimensional spectrum of the refractive index fluctuations. Within the classical framework of isotropic and homogeneous turbulence theory, it is possible to relate this three-dimensional spectrum to global parameters such as the refractivity structure constant $\mathrm{C}_{n}^{2}$. However, the aspect sensitivity of VHF radar echoes [Rottger et al., 1978; Green et al., 1979; Rottger et al., 1981; Tsuda et al., 1985] shows that the three-dimensional spectrum is not isotropic for a wavelength of $\approx 3 \mathrm{~m}$. Thus, the relationship between the radar echoes and the local structure of turbulence is worth studying with the help of in situ measurements. The results of such a direct comparison experiment (the first to our knowledge) are reported here, along with a tentative theoretical interpretation.

A theoretical attempt to relate the angular variation of the radar echoes to the local structure of the scattering medium was made by Briggs and Vincent [1973]. This first approach 


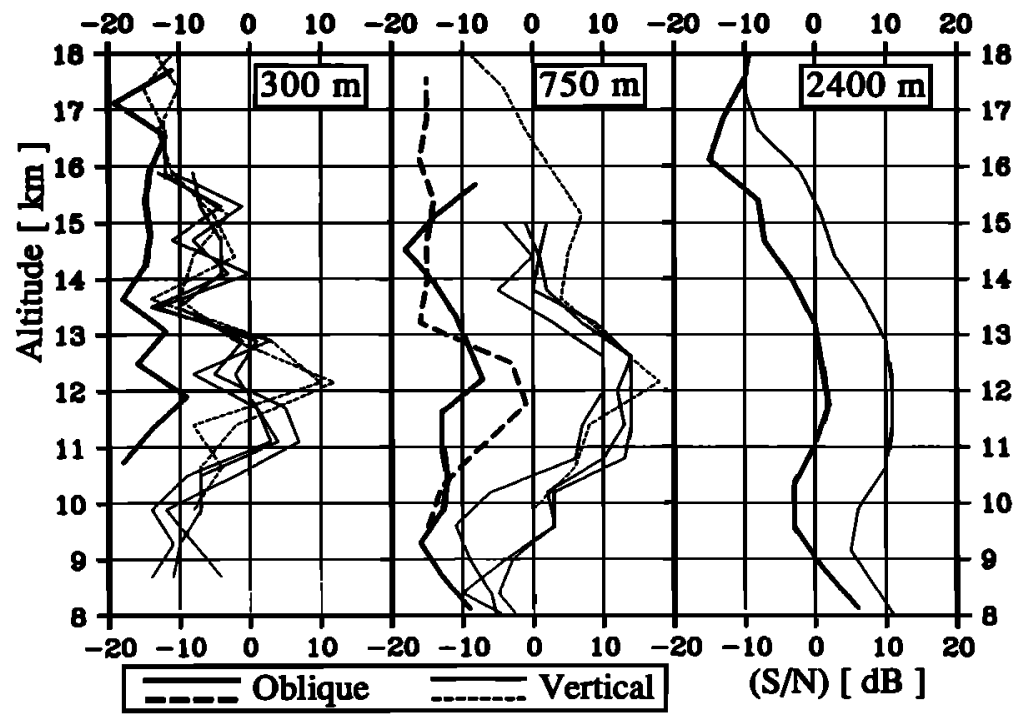

Fig. 1. Vertical profiles of measured signal to noise ratio $\mathrm{S} / \mathrm{N}$ for both vertical and oblique line of sight. The three available range resolutions are plotted: $\Delta r=300 \mathrm{~m}$ (left panel), $\Delta r=750 \mathrm{~m}$ (middle) and $\Delta \mathrm{r}=2400 \mathrm{~m}$ (right). Oblique echoes (bold lines) are about $10 \mathrm{~dB}$ lower than vertical ones, especially in the stratosphere. Continuous and dashed lines indicate measurements with different sampling altitudes. The strength of the echoes seems to depend strongly on the precise altitude of measurement. There is also a noticeable time variability for a given sampling point.

assumed that all inhomogeneities have the same size, the same amplitude and an anisotropic gaussian shape. Recently, Hocking [1987] extended this approach for the case of a continuous distribution of sizes and amplitudes. A convenient theoretical framework for such studies can be found in the work of Doviak and Zmic' [1984] because these authors did not make any assumptions about the spectrum (and thus the shape) of the scatterers and because they included the effect of the curvature of the wave front.

In section 2, we give a rapid overview of the experiment and of the main characteristics of the instruments used for both remote sensing and in situ measurements. Typical results obtained by the instruments are presented.

In section 3, we describe the quantities measured by each type of instrument, and give the relationships between them under the assumption of isotropy. We then present a new physical interpretation of the radar observed anisotropy, which stems from recent theoretical approaches of the turbulence in a stratified medium. These approaches lead to turbulent fluctuation spectra involving two terms corresponding respectively to inertial (isotropic) turbulence and buoyancy affected (presumably anisotropic) turbulence. Presently available theories do not give any account for this anisotropy. We thus introduce a semiquantitative model for the threedimensional anisotropic turbulent spectrum and we use it to reconstruct the observed radar profiles, for both the oblique and vertical directions, starting with the high-resolution balloon measurements.

In section 4 , we try to interpret the obtained results, and compare them with previous models for radar reflectivity, based either on a statistical model of the turbulence (oblique measurements) or on a "Fresnel reflection" model (vertical measurements). Our tentative reconstruction leads to puzzling results:

1. In the oblique direction, where the classical theory is expected to apply, the shape and dynamic ranges of the echo profiles are poorly reproduced.

2. In the vertical direction, where we use our new assumptions, the shape and dynamic ranges are conveniently reproduced.

3. In both cases, the predicted levels are strongly overestimated. However, if we assume that the buoyancyaffected spectrum is isotropic (while the radar tells us that it is not), we obtain (by chance?) the correct level in the vertical direction.

We then examine the various experimental and theoretical uncertainties, and conclude that the discrepancy is real. After a discussion of the results of this work and a comparison with previous models, we draw some conclusions and call for further studies of this problem.

\section{THE INSTRUMENTS}

\subsection{The radar}

The radar used for this comparison was the stratospheretroposphere (ST) VHF radar "Provence" of the University of Toulon located near Termes d'Armagnac (southwest of France: $0^{\circ} 01^{\prime} \mathrm{W}, 43^{\circ} 40^{\prime} \mathrm{N}$, altitude $116 \mathrm{~m}$ ). Its main characteristics are as follows: frequency (wavelength), $47.78 \mathrm{MHz}\left(\lambda_{\mathrm{R}}=\right.$ $6.28 \mathrm{~m}$ ); power attenuation, $\alpha=0.35$; radiated peak power, $\alpha P_{t}=10 \mathrm{~kW}$; geometric area, $A_{g}=54.4 \times 47.1=2563 \mathrm{~m}^{2}$; 


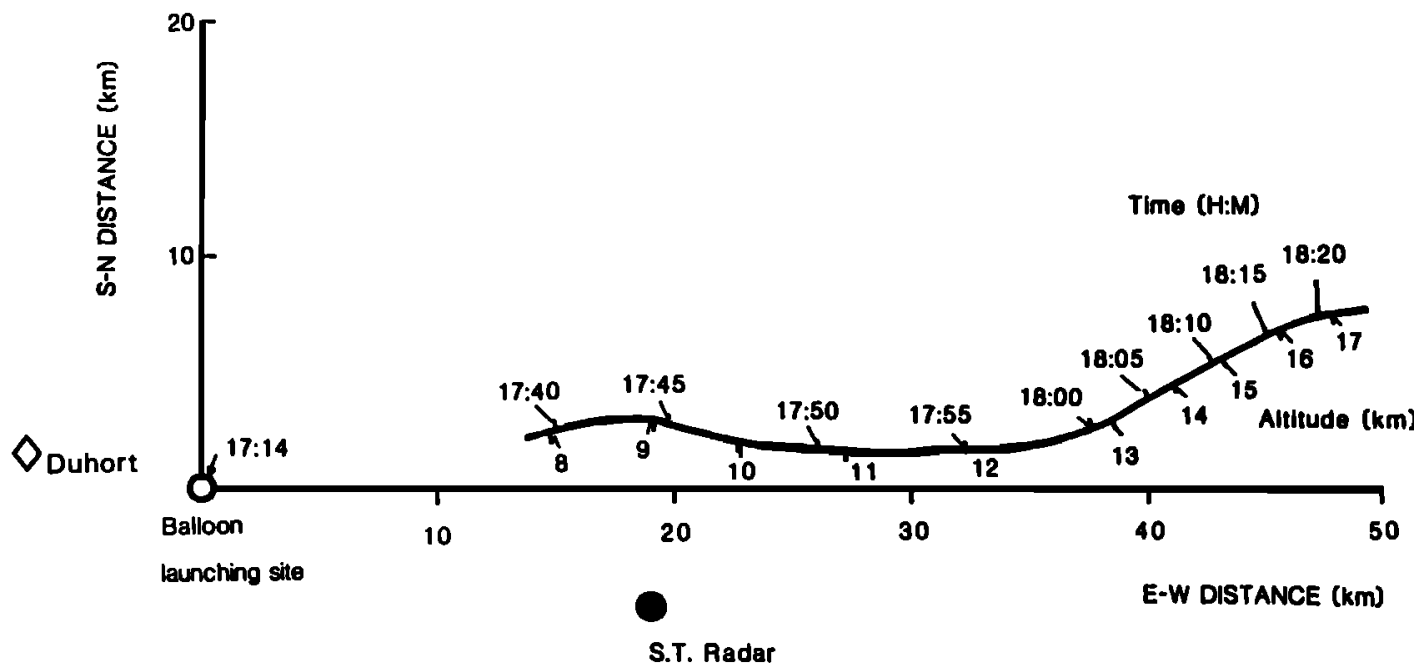

Fig. 2. Ground projection of the trajectory of the instrumented balloon. This trajectory is labeled with the universal time (above) and with the altitude of the balloon (below). The position of the ST radar is indicated with a solid circle and the meteorological station of Duhort with a diamond.

zenith angle, $0^{\circ}$ (vertical) and $14.5^{\circ}$ (oblique toward west); and range resolution used, $\Delta r=300 \mathrm{~m}, 750 \mathrm{~m}$ and $2400 \mathrm{~m}$.

The coefficient $\alpha$ includes coaxial line attenuation, VSWR (voltage standing wave ratio) effect and estimated losses in radiating elements. The receiver used order 2 Bessel filters with a bandwidth matched for each pulse length. The vertical profiles of signal to noise ratio $(S / N)$ were measured for the three available resolutions and in both vertical and oblique directions. Typical profiles are shown on Figure 1. As usual, with this type of measurements, the vertical echoes are roughly $10 \mathrm{~dB}$ stronger than oblique ones in the stratosphere [Rottger et al., 1978]. High and medium resolution profiles ( 300 and $750 \mathrm{~m}$ ) are quite variable in time (see Figures 1 and 7) and their detailed structure may change drastically, depending on the position of the sampling points.

\subsection{The instrumented balloon}

An instrumented balloon was launched from the Centre National dEtudes Spatiales (CNES) base of Aire sur l'Adour $\left(0^{\circ} 15^{\circ} \mathrm{W}, 43^{\circ} 42 \mathrm{~N}\right.$, altitude $\left.76 \mathrm{~m}\right)$ on May 4,1984 at $1714 \mathrm{UT}$. The ground projection of its trajectory, during its ascent, is shown on Figure 2 along with the location of the ST radar and of the radio-sounding station of Duhort $\left(0^{\circ} 20 \mathrm{~W}, 43^{\circ} 43^{\prime} \mathrm{N}\right.$, altitude $140 \mathrm{~m}$ ). The minimal horizontal distance between the balloon and the radar was $\approx 8 \mathrm{~km}$ (north) at an altitude of $=9 \mathrm{~km}$. The instrumented gondola was hung $140 \mathrm{~m}$ below a $5000-\mathrm{m}^{3}$ zero-pressure balloon. Taking into account the ascent velocity and the known shear statistics, this distance is sufficient to avoid any contamination of measurements by the balloon wake [Barat et al., 1984].

The instruments included a two-axis (horizontal and vertical) ionic anemometer [Barat, 1982], a pressure sensor and two thermistors in order to provide coarse and fine temperature measurements. The sampling frequency was $64 \mathrm{~Hz}$. The fine temperature sensor was designed to operate under stratospheric conditions; thus it was saturated for altitudes lower than $\approx 8 \mathrm{~km}$. The altitude of the gondola was precisely determined by integration of the hydrostatic equation. Extensive spectral analysis of vertical velocity and temperature data was performed. The calculated one-dimensional spectra are considered as longitudinal because the relative horizontal velocity at the gondola level is always small compared to the vertical one during the ascent. Transverse spectra of the horizontal velocity could not be considered because the upwind orientation of the horizontal anemometer was not operational during the rapid ascent $\left(=5 \mathrm{~m} \mathrm{~s}^{-1}\right.$ in the troposphere and $\approx 3$ $\mathrm{m} \mathrm{s}^{-1}$ in the stratosphere).

The scale range under study is slightly variable with the balloon velocity, but always includes the half radar wavelength $\lambda_{R} / 2=3.14 \mathrm{~m}$. Furthermore, the balloon itself being insensitive to such small-scale velocity fluctuations, the anemometric data at these scales only correspond to atmospheric fluctuations at the gondola level. The temperature spectra are corrected for the time response of the sensor. This density dependent time constant is in the range $0.3-0.5 \mathrm{~s}$, thus affecting scales smaller than $5-10 \mathrm{~m}$. The correction, when applied to the high-frequency white noise of the sensor produces a spurious $(+2)$ slope. Typical vertical velocity (W) and temperature (T) vertical spectra are shown on Figure 3. These spectra are obtained within a data section of about $1 \mathrm{~km}$ just above the tropopause. An inertial subrange (with a slope close to $-5 / 3$ ) is observed on both spectra for scales smaller than about $10 \mathrm{~m}$. At larger scales, temperature and velocity spectra increase with a steeper slope (-3) because of stratification effects. The physical nature of the fluctuations leading to such slope is still uncertain. It can be seen as an extension toward small scales of a "universal" gravity wave spectrum, first introduced for the atmosphere by VanZandt [1982]. Currently available gravity wave spectrum models 


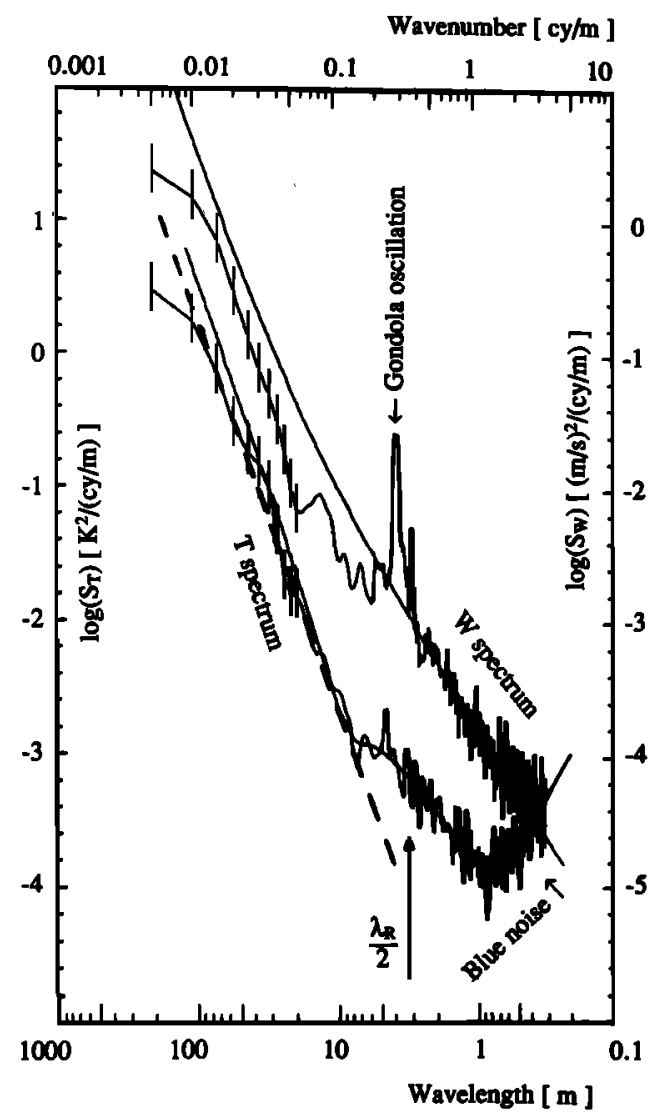

Fig. 3. Temperature (lower curve and left vertical axis) and vertical velocity (upper curve and right vertical axis) vertical spectra as measured by the instrumented balloon in a $1-\mathrm{km}$ slab of the stratosphere, just above the troposphere. The statistical 90\% confidence level (constant in log-log coordinates) is indicated on the left part of each spectrum. The dashed line, with a -3 slope, corresponds to the prediction of a gravity wave model [Sidi et al., 1988] for the vertical velocity spectrum. The two smooth curves show a theoretical fit from the turbulent theory [Dalaudier and Sidi, 1987] used in the present work. The sharp peak in the velocity spectrum corresponds to the gondola oscillation. Two regions can be distinguished in both spectra: an "inertial" subrange (for small scales) with a spectral slope close to $-5 / 3$ and a "buoyancy" subrange (for large scales) with a spectral slope close to -3 . The half radar wavelength is clearly in the " $s-5 / 3$ " part of the temperature spectrum and well above the "blue noise" level (the rightmost part with a slope of +2 ).

admit a $\mathbf{- 3}$ slope in their high wave number limit [Dewan and Good, 1986; Smith et al., 1987; Sidi et al., 1988]. It can also be interpreted as a "buoyancy range" of the turbulence [Dalaudier and Sidi, 1987]. The conspicuous peak observed on the velocity spectrum corresponds to a mechanical oscillation of the gondola and must be ignored.

\subsection{The rawinsondes}

The experiment took place during the meteorological campaign "Front 84" and, thus, many soundings of the atmosphere were performed by standard rawinsondes launched from the nearby mobile station of Duhort (see Figure 2). These soundings provided vertical profiles of wind and temperature along with humidity (in the troposphere) with a resolution of $\approx 30 \mathrm{~m}$. These profiles will be used for comparison with simultaneous radar measurements.

\section{COMPARISON BETWEEN IN SITU AND REMOTE MEASUREMENTS}

Because the two kinds of instruments do not measure the same physical quantity, in situ measurements cannot be compared directly with the results of remote sensing.

\subsection{The ST radar}

Under clear air conditions, the reflectivity of the atmosphere produced by density (or temperature) and humidity fluctuations can be measured by the radar. The backscattered signal from a given volume is directly proportional to the three-dimensional spectrum of refractive index fluctuations [Tatarski, 1961; Ottersten, 1969b] at the radar half wavelength, along its line of sight. The spatial distribution and the shape of the density inhomogeneities (scatterers) can range from very ordered to completely random. For example, Gage and Balsley [1980] distinguished Fresnel reflection, Fresnel scattering, and turbulent scattering (anisotropic or isotropic). Other mechanisms were also proposed such as the "diffuse reflection" [Rottger, 1980] in which horizontal inhomogeneities (laminae) are "corrugated" by turbulence. However, for all the mechanisms that assume a random distribution of scatterers, the properties of the medium can always be described by a three-dimensional spectrum. With this formulation, there is no need for a special expression for the case of a stratified medium; the aspect sensitivity of the radar is simply interpreted as resulting from the anisotropy of the threedimensional temperature spectrum. This formulation, using a three-dimensional spectrum, cannot account for discrete reflection phenomena sometimes observed in the vertical direction. However, this kind of echo is rather exceptional: Sheen et al. [1985] report 10\% of partial reflections for a range resolution of $300 \mathrm{~m}$, and argue that longer pulses should reduce this proportion. Furthermore, they affect only one gate and can be easily identified. In the other cases, the anisotropic inhomogeneities responsible for the radar echoes "fill" the observed volume, leading to a backscattered power (roughly) proportional to the pulse length [Green and Gage, 1985]. The possible intermittent structure of the fluctuation field ("filling factor") is readily taken into account through the global level of the three-dimensional spectrum.

Taking into account the finite volume of measurement and the sphericity of the wave surface, Doviak and Zmic' [1984] showed that this three-dimensional spectrum should be convolved with a "spectral sampling function" (SSF) depending on the range resolution and the radar characteristics. This convolution procedure produces a noticeable effect only if 
the variations of the three-dimensional spectrum are important inside the spectral volume sampled by the SSF. For the present study, the range resolution used is sufficiently large in order to ignore its effects on the spectrum (variation less than 1\%). Regarding the lateral extent of the scattering volume (the lobe width is here $\approx 5.2^{\circ}$ ), its effect can be important if the three-dimensional spectrum is highly anisotropic with a characteristic width smaller than (or comparable to) the lobe angular resolution. The observed angular extent of the anisotropic echo around the vertical (see, for exemple, Tsuda et al. [1985]) is precisely of this order. An estimate of the combined effect of the lobe width and of the spectrum anisotropy is given in the appendix.

Except for this angular convolution effect, the formulas used to convert the three-dimensional spectrum of temperature fluctuations into signal to noise ratio $(S / N)$, assuming that cosmic noise is dominant at this frequency, are [VanZandt et al., 1978; Ottersten, 1969a, b]

$$
\begin{aligned}
S / N=\frac{4}{9} \frac{\alpha P_{t} A_{g}}{c k_{B} T_{N}} & \left(\frac{\Delta r}{r}\right)^{2}\left(4 \pi k_{R}^{4}\right) N_{C I} \phi_{n}\left(2 k_{R}\right) \\
\phi_{n} & =\left(\frac{g M}{T N_{B V}^{2}}\right)^{2} \phi_{T} \\
\phi_{n} & =\left(\frac{Q_{p}}{T}\right)^{2} \phi_{T}
\end{aligned}
$$

where (1b) gives the general case and (1c) gives the "dry air" case, with

$\phi_{n} \quad$ three-dimensional spectrum of refractive index fluctuations;

$\phi_{\mathrm{T}}$ three-dimensional spectrum of temperature fluctuations;

M generalized gradient of potential refractive index [Ottersten, 1969a];

$\mathrm{N}_{B V}$ Brunt-VHistlly frequency;

$Q$ conversion factor between density and "dry" refractive index $\left(=2.23 \times 10^{-4} \mathrm{~m}^{3} \mathrm{~kg}^{-1}\right)$;

g gravity acceleration;

P air density;

T air temperature;

$k_{R}$ radar wave vector (with modulus $k_{R}=2 \pi / \lambda_{R}$ );

$\mathrm{NCI}$ number of coherent integrations used to make the measurement;

$\Delta r \quad$ range resolution;

r radial distance;

c velocity of light;

kB Boltzmann constant;

$\mathrm{T}_{\mathrm{N}}$ cosmic noise temperature $(\approx 6700 \mathrm{~K})$.

The cosmic noise temperature $\mathrm{T}_{\mathrm{N}}$ was determined for each measurement by logarithmic interpolation between maps of the radio sky at three frequencies: $38 \mathrm{MHz}$ [Blythe, 1957], 64 $\mathrm{MHz}$ [Hey et al., 1947] and $150 \mathrm{MHz}$ [Landecker and Wielebinsky, 1970]. For the sake of simplicity, we used a constant value; the systematic error associated with the variation of $T_{N}$ during the experiment is given in section 4.3.
In the "dry air" case, we have used a direct conversion between density and refractive index through the factor $Q=7.76 \times 10^{-7} \mathrm{R}$ (taking into account the conversion of pressure from millibars to pascals), $R=287 \mathrm{~J} \mathrm{~K}^{-1} \mathrm{~kg}^{-1}$ being the gas constant for air. This conversion formula will be used above $8 \mathrm{~km}$ where the effect of humidity is negligible.

\subsection{In situ measurements}

Measurements made by regularly sampling the local medium properties, along approximately straight and uniform trajectories, lead (with the help of the Taylor hypothesis) to a one-dimensional spectrum of these quantities. The onedimensional spectrum $S$ corresponds to an integration (in wave number space) of the three-dimensional spectrum $\phi$ along two planes perpendicular to the velocity direction, symmetric with respect to the origin, their distance to the origin being the observed wave number. For example, if the instrument velocity is vertical, the one-dimensional spectrum is $\left(\mathbf{k}_{\mathrm{x}}, \mathbf{k}_{\mathbf{y}}\right.$ and $k_{2}$ being the components of $k$ )

$S\left(k_{z}\right)=\iint \phi\left(k_{x}, k_{y}, k_{z}\right) d k_{x} d k_{y}+\iint \phi\left(k_{x}, k_{y},-k_{z}\right) d k_{x} d k_{y}$

$\mathbf{k}_{\mathbf{z}}$ being kept constant during the integration. In general, it is not possible to deduce the value of the three-dimensional spectrum at a given point from the knowledge of the onedimensional spectrum $S(\mathrm{k})$. Conversely, the knowledge of the three-dimensional spectrum $\phi$, limited to only one wave number modulus (as with ST radar measurements) does not allow us to deduce, in general, the one-dimensional spectrum.

\subsection{Isotropic conversion formulas}

However, if the three-dimensional spectrum is isotropic, that is, depends only on the modulus of $\mathbf{k}$, conversion between three-dimensional and one-dimensional spectra becomes possible. The relation is [Monin and Yaglom, 1975]

$$
\frac{d S}{d k}=-4 \pi k \phi(k)
$$

for the spectrum of a scalar quantity. Another spectral representation, commonly used in theoretical studies, is the scalar spectrum E. It corresponds to an integration of the threedimensional spectrum on a spherical shell of radius $k$. This definition shows that, in the isotropic case

$$
E=4 \pi k^{2} \phi(k)
$$

Usually, the one-dimensional spectrum can be expressed as a power law (at least in some vicinity of $\mathbf{k}$ ). If the spectral index is $p$, conversion formulas become

$$
E=-p S=4 \pi k^{2} \phi(k)
$$

Thus, in the isotropic case, the in situ measurement of a one-dimensional temperature spectrum allows us to reconstruct the vertical profile of $S / N$ ratio observed with an ST radar. We will present our results in the form of $S / N$ profiles instead of the more classical $C_{n}^{2}$ profiles, because this parameter can be defined unambiguously only in the case of inertial and isotropic turbulence. Experimental evidence points against 


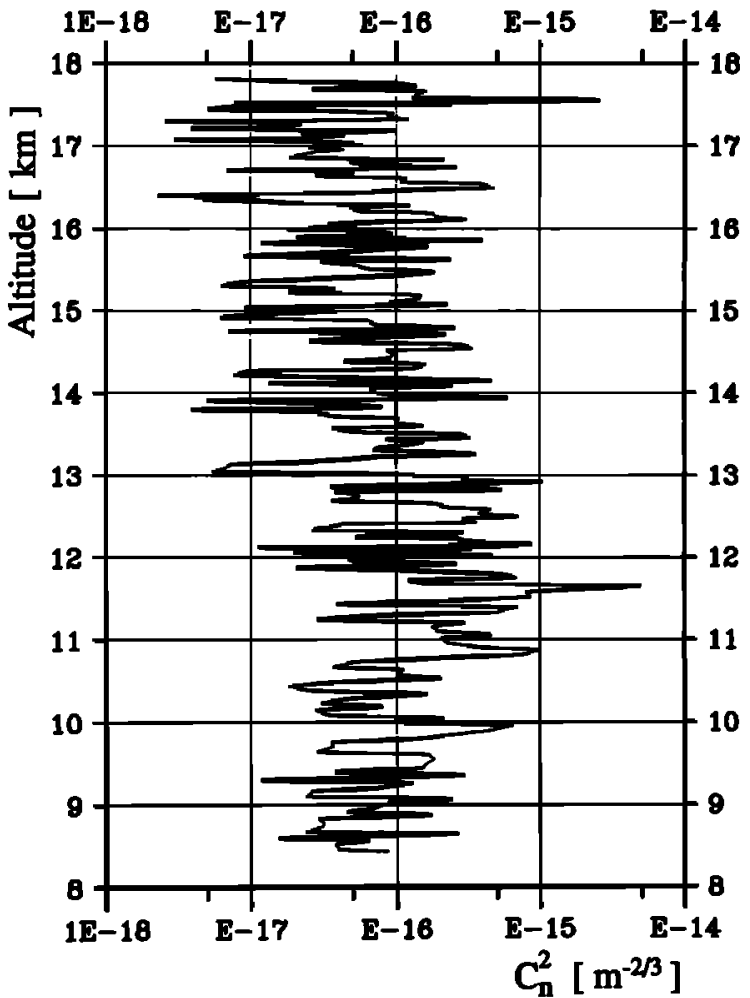

Fig. 4. Vertical profile of $\mathrm{C}_{\mathbf{n}}^{2}$ deduced from in situ measurements of the temperature spectra at $3.14 \mathrm{~m}$ (the half radar wavelength). The conversion formulas assume that the humidity is negligible and that the spectrum is isotropic with a $-5 / 3$ slope. Each estimate corresponds to $10 \mathrm{~s}$ of measurement $(\approx 30$ to $50 \mathrm{~m}$ ). The local dynamical range is larger than one decade everywhere, thus indicating that the noise level does not significantly affect the estimation.

such a simple hypothesis, especially for the comparison with vertical radar measurements.

However, in order to allow comparison with typical published values, we have plotted on Figure 4 a profile of $C_{n}^{2}$ deduced from the observed one-dimensional temperature spectra (for $k=2 \mathrm{rad} \mathrm{m}^{-1}$ ) through the inertial and isotropic

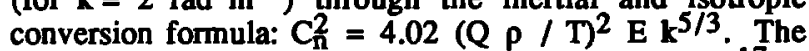
reported values are approximately in the range $10^{-17}$ to $5 \times 10^{-16} \mathrm{~m}^{-2 / 3}$ in the stratosphere with a strong increase (values above $10^{-15} \mathrm{~m}^{-2 / 3}$ ) just above the tropopause, probably associated with the jet (about $35 \mathrm{~m} \mathrm{~s}^{-1}$ ) observed around $10.5 \mathrm{~km}$. Such values of the refractive index structure parameter can be considered as large, when compared with other published data [Azouit et al., 1980], but are probably representative of disturbed meteorological conditions.

\subsection{Characteristics of the vertical echoes}

It is well known that VHF radars obtain stronger echoes from the vertical direction than from the off-vertical one (aspect sensitivity, for example, see Tsuda et al. [1985]). In order to interpret this angular variation, Doviak and Zmic' [1984] proposed that the three-dimensional spectrum can be approximated as the sum of an isotropic part and of a strongly anisotropic one with a sharp maximum in the vertical direction. Estimates of the anisotropy were made by Waterman et al. [1985] with the sounding system (SOUSY) radar (50 MHz) at Arecibo. Assuming an ellipsoidal geometry for the autocorrelation function, they found that, for most altitudes, the ratio of horizontal to vertical correlation length is larger than 5.

Another characteristic of the vertical echoes is their strong sensitivity to the medium stability, measured by the BruntVuisala frequency NBV [Tsuda et al., 1985]. Gage et al. [1985] proposed a semiempirical model for vertical echoes where the reflectivity is proportional to $\mathrm{N}_{\mathrm{B}}^{4}$. This model assumes that the inhomogeneities, responsible for the vertical echoes, result from the vertical displacement of fluid particles in the stably stratified fluid. Their spectrum is thus proportional to the squared vertical gradient of potential refractive index $\mathrm{M}=(\mathrm{Q} \rho / \mathrm{g}) \mathrm{N}_{\mathrm{BV}}$. This model reproduces very well (within an adjusted constant) the observed vertical profile of S/N. A recent experimental study by Tsuda et al. [1988] also concludes that the vertical echo power and $\mathbf{M}^{2}$ are proportional.

Finally, the aspect sensitivity is known to be very dependent on the radar wavelength. For example, Balsley and Peterson [1981], using the Chatanika radar with a 23-cm wavelength reported the absence of aspect sensitivity. This observation constitutes an indication that the anisotropic part of the spectrum, responsible for the aspect sensitivity, decreases faster than the isotropic part as the wave number is increased.

\subsection{Approach used for vertical and oblique reconstruction}

It has been suggested that the physical process responsible for the radar echoes in the vertical direction could be a field of small-scale random gravity waves [VanZandt and Vincent, 1983; Gage et al., 1985]. Besides the fact that gravity waves (a linear phenomenon) probably cannot persist in the same scale range (here $\approx 3 \mathrm{~m}$ ) as the observed turbulent (fully nonlinear) fluctuations, this interpretation is incompatible with the in situ observed energy level of the vertical velocity onedimensional spectrum. For scales smaller than $\approx 10 \mathrm{~m}$, the observed spectrum is at least an order of magnitude above the predicted energy level for a saturated wave field. Figure 3 shows the vertical velocity spectrum (dashed line) predicted by the model of Sidi et al. [1988]. The parameters of this model were fitted to the same in situ data set for scales larger than $\approx 200 \mathrm{~m}$, but the discussed result is typical and any available gravity wave model would have lead to a similar conclusion, owing to the strong anisotropy assumed for the velocity field.

Other theories are in competition in order to account for in situ measured one-dimensional spectra. One [Holloway, 1983] is derived from a closure approach intended to describe, in a single framework, gravity waves, vortical modes and turbulent motions. Another [Dalaudier and Sidi, 1987] follows the approach of Lumley [1964] and Weinstock [1985] in order to generalize the classical turbulence theory in the presence of a stable stratification. While the two approaches are rather 
different, they both agree on the following description of the temperature spectrum as a superposition of two components. The first one corresponds to the turbulent advection of fluid particles and exhibits the well known $k^{-5 / 3}$ power law in the inertial subrange (ISR) if it exists. The second one corresponds to the vertical displacement of fluid particles in the stratified background. It dominates the scalar spectrum at low wave numbers, in the so-called "buoyancy subrange" (BSR) with a universal energy level scaling like $\mathrm{N}_{\mathrm{B}}^{2} \mathrm{k}^{-3}$ (the two spectral domains are observed in Figure 3). Both theoretical considerations and experimental evidence point toward the anisotropy of the temperature spectrum, at least in the BSR. For clarity, this anisotropic component, present at all scales, will be termed hereafter "the BSR spectral component", even if we consider it at scales much shorter than those of the BSR.

Thus, the physical interpretation used below, in order to reconstruct the radar signal, is similar to the one used by Doviak and Zrnic' [1984]. The oblique signal is supposed to result from the inertial and isotropic spectral component, the level of which is obtained by in situ temperature measurements. The vertical signal is supposed to result from the three-dimensional anisotropic BSR component. Owing to its anisotropy, this spectrum dominates the inertial one for directions close to the vertical (in agreement with radar observations). This physical interpretation allows us to understand in a natural way the dependency of vertical echoes upon the local stability and the disappearance of the aspect sensitivity for smaller wavelengths (because of its -3 slope, the BSR spectrum decreases faster than the inertial one for high wave numbers).

\subsection{Effect of anisotropy}

In order to investigate the effect of an assumed anisotropy of the three-dimensional spectrum on both balloon and radar measurements, we have made some analytical calculations with a simple model of anisotropy (see appendix). In this model, the anisotropy is characterized by the (constant) ratio $\beta$ between horizontal and vertical correlation lengths. If the scalar spectrum $E$ presents a spectral slope $p$, the observed onedimensional vertical spectrum is then $\mathrm{S} \approx \mathrm{E}$ for the strongly anisotropic case and $S=-E / p$ for the isotropic one. The ratio between the two extreme cases ( $-p)$ is quite small compared to the range of "natural variability" of the observed spectral level. Thus, it is not possible to infer the amount of anisotropy from the in situ observed one-dimensional spectral level in the BSR. By contrast, the vertical three-dimensional spectrum $\phi\left(0,0, \mathbf{k}_{\mathrm{z}}\right)=-(\mathrm{p} A) /(4 \pi \beta)\left(\mathbf{k}_{2} / \beta\right)^{(\mathbf{p}-2)}$ changes very rapidly with $\beta$. Even if this variation is limited, for radar measurements, by the finite size of the lobe (as shown in the appendix), the increase for a typical value of the anisotropy ( $\beta$ $\geq 5$ ) is larger than 65 . Furthermore, the angular variation is very rapid and the ratio between the anisotropic contribution to vertical and oblique measurements is expected to be always larger than 10. This explains why the radar can see the anisotropic spectrum in the vertical direction but not in the oblique one, while for in situ measurements, the isotropic spectrum dominates for the same scale.

However, in the following comparison, we will use only isotropic relationships. This seems quite reasonable for oblique measurements, as the three-dimensional spectrum is supposed to correspond to the presumably isotropic inertial domain of turbulence. For the vertical direction, however, the spectrum is suspected to be anisotropic, but the exact nature and amount of this anisotropy are unknown and the in situ measurements cannot help in determining its characteristics. Thus the comparisons will be made by using isotropic formulas, keeping in mind that this will produce a strong underestimation of the predicted $S / N$ ratio in the vertical direction.

\subsection{Oblique comparison}

The spectral density of the temperature fluctuations at half radar wavelength $\left(k=2 \mathrm{rad} \mathrm{m}^{-1}\right)$ is estimated from smoothed periodograms computed every 10 -s data section. The associated noise equivalent bandwidth $\left(\Delta \mathbf{k}=0.8 \mathrm{rad} \mathrm{m} \mathrm{m}^{-1}\right)$ ensures a sufficiently small statistical confidence interval. Depending on the balloon ascent velocity, each data section corresponds to a layer thickness $\approx 50 \mathrm{~m}$ in the troposphere and $\approx 30 \mathrm{~m}$ in the stratosphere. This vertical resolution is far better than the smallest range resolution of the radar. The spectrum is then converted from one-dimensional to three-dimensional with the help of (5) assuming $p=-5 / 3$ and the height series is smoothed with the radar height sampling function. This function is taken to be triangular with a full width at half maximum equal to the range resolution considered reduced by the geometrical factor $\cos \left(15^{\circ}\right)$. The profile of the $\mathrm{S} / \mathrm{N}$ ratio is then reconstructed using (1) with the same sampling altitude as that of the radar. The result is plotted, along with the corresponding radar measurements, on Figure 5 for the three range resolutions available.

As can be seen, this reconstruction is quite disappointing; some outstanding problems can be noted: (1) The general level is overestimated by a factor $\approx 30(15 \mathrm{~dB})$. (2) The dynamic range of the reconstructed signal is too low compared to the observed one. (3) The shape of the observed profiles is poorly reproduced.

All these discrepancies will be discussed below, after the vertical comparison.

\subsection{Vertical comparison}

As explained above, in situ measurements of spectral levels cannot be used to reconstruct vertical radar profiles. Instead, theoretical spectral levels are used with only an adjustment to the local mean temperature and Brunt-V tisals (B-V) frequency. Conversion between scalar and three-dimensional spectra is made through the isotropic relation (4). Then, the reconstruction process is similar to the one used for oblique measurements, except for geometrical factors. The proposed theoretical scalar temperature spectrum is [Sidi and Dalaudier, 1988]

$$
E_{T}=2 B_{a} \frac{T^{2} N_{B V}^{4}}{g^{2} k^{3}}
$$

where $B_{a}$ is the Batchelor constant $\left(B_{a}-1.16\right)$. With this spectrum, the reconstructed signal will be proportional to $\mathrm{N}_{\mathrm{B}}^{4} \mathrm{v}$, as in the model of Gage et al. [1985], but there is no parameter adjustment before comparison. The reconstructed 


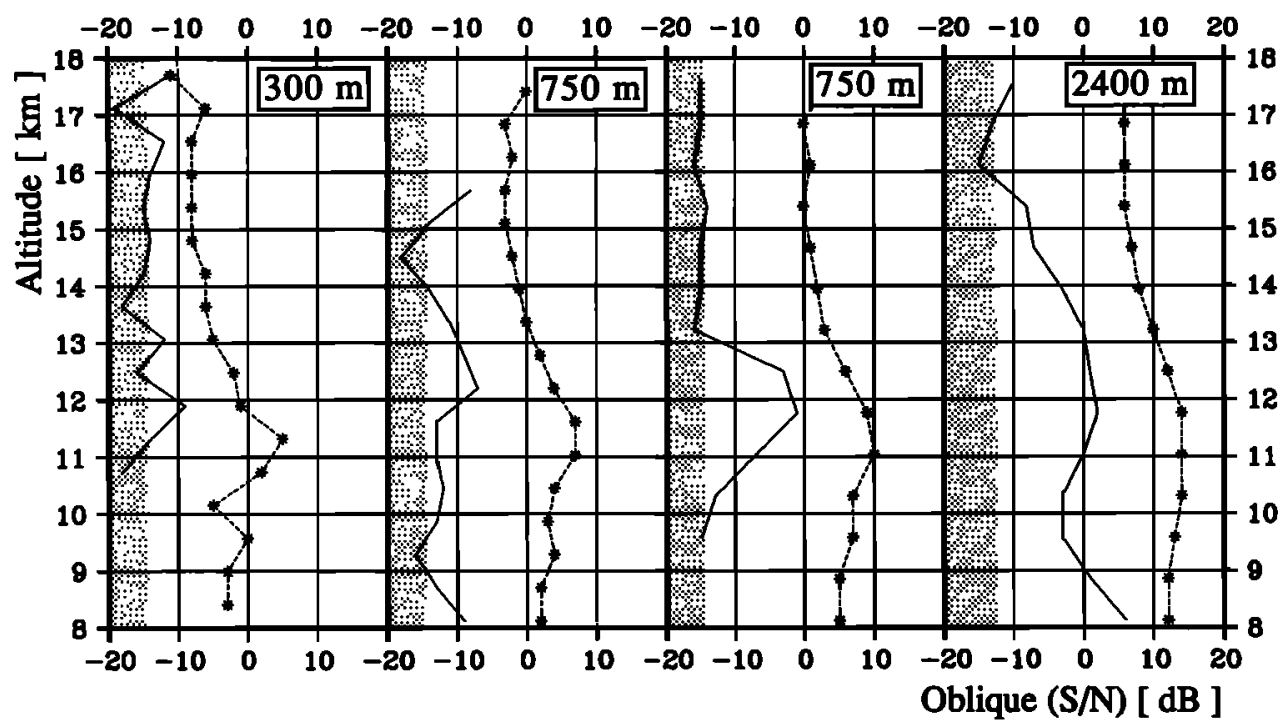

Fig. 5. Vertical profiles of measured (solid line) and reconstructed (dashed line with asterisks) signal to noise ratio (S/N) for oblique radar measurements. The regions where the radar echoes cannot be distinguished from the noise are shaded. The reconstruction process uses the $C_{n}^{2}$ shown on Figure 4 and a triangular convolution window with a full width at half maximum equal to the corresponding radar resolution. The four panels correspond respectively to range resolutions $\Delta \mathrm{r}=300 \mathrm{~m}$ (left panel), $\Delta \mathrm{r}=750 \mathrm{~m}$ (the two central panels) and $\Delta r=2400 \mathrm{~m}$ (right). The comparison shows that the shape and the dynamic range are poorly reproduced. Furthermore, the level of the reconstructed profile is 10 to $15 \mathrm{~dB}$ above the measured one.

profile is plotted, along with the corresponding radar measurements on Figure 6 for the three range resolutions available.

This reconstruction is rather good, especially for medium and coarse resolutions: (1) The general level is close to the observed one (slightly underestimated). (2) The dynamic range of the reconstructed signal is comparable, or slightly lower, than the observed one. (3) The detailed shape of the profiles are very similar, except for some "high-resolution" profiles.

Because only moderate resolution temperature profiles are needed for this vertical reconstruction, it is possible to use radio-sounding data. Four reconstructions were performed at different times and days, each being compared with three radar profiles, separated by about half an hour, corresponding approximately to the beginning, the middle and the end of the radio sounding. The vertical resolution of the sounding was $\approx 32 \mathrm{~m}$ while the radar range resolution was always $750 \mathrm{~m}$. The results of these reconstructions, including the effect of humidity in the troposphere, are shown on Figure 7 along with the radar measurements. Here again, the reconstructed profiles are very similar to the measured ones (taking into account the time variability of this kind of measurements) and the same remarks as for Figure 6 can be made. Under $8 \mathrm{~km}$, the reconstructed profile of $S / N$ sometimes presents sharp decreases that are not observed by the radar. These localized discrepancies are artifacts linked with the low local value of the humidity (under the detection level of the rawinsonde equipment).

\section{INTERPRETATION AND DISCUSSION}

\subsection{The "successful" vertical reconstruction}

The detailed shape agreement observed on Figure 6 constitutes a confirmation of earlier results [Gage et al., 1985; Tsuda et al., 1988] showing that the part of the threedimensional spectrum responsible for the vertical echoes is proportional to $\mathrm{N}_{\mathrm{BV}}^{4}$. A natural interpretation of this relation, as pointed out above, is that these temperature fluctuations result mainly from the vertical (and adiabatic) displacement of fluid particles in the stably stratified background.

The fact that the medium and coarse resolution profiles are better reproduced than the high-resolution ones can be easily understood as a consequence of the finite horizontal and temporal extent of vertical structures. This extent is expected to be larger for larger vertical scales and should be compared with the space and time distance between the measurements of the two instruments.

The dynamic range of the reproduced profiles is comparable to, but slightly lower than the observed one. This "statistical" property depends on the value of the exponent of $\mathrm{NBV}_{\mathrm{BV}}$ in (6). The general agreement shows that 4 is a good value, and a very simple mechanism can be proposed to slightly increase the dynamic range. It is known that the three-dimensional spectrum responsible for vertical echoes is anisotropic. This anisotropy is presumably dependent on the local stability, a stronger stratification leading to a higher anisotropy and thus 


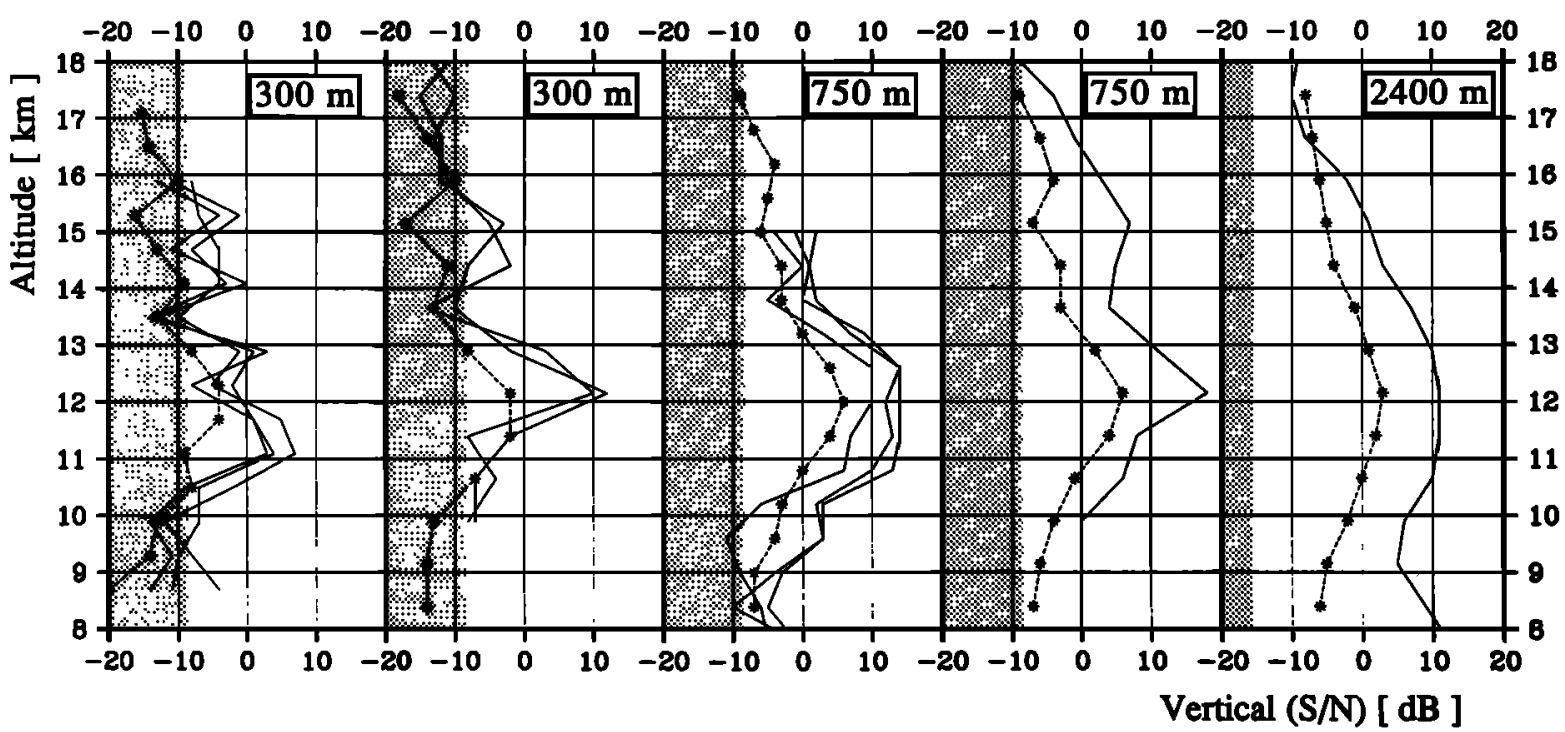

Fig. 6. Vertical profiles of measured (solid line) and reconstructed (dashed line with asterisks) signal to noise ratio (S/N) for vertical radar measurements. The regions where the radar echoes cannot be distinguished from the noise are shaded. The reconstruction process is based only on the in-situ measurements of the vertical gradient of potential temperature and uses a triangular convolution window as for the oblique direction. The range resolutions are, respectively from left to right, $\Delta r=300,300,750,750$ and $2400 \mathrm{~m}$. The shape is now well reproduced, particularly for medium and coarse resolution. The dynamic range is also quite satisfactory, although slightly smaller than the observed one. The mean level of the reconstructed profile is now smaller than the measured one.

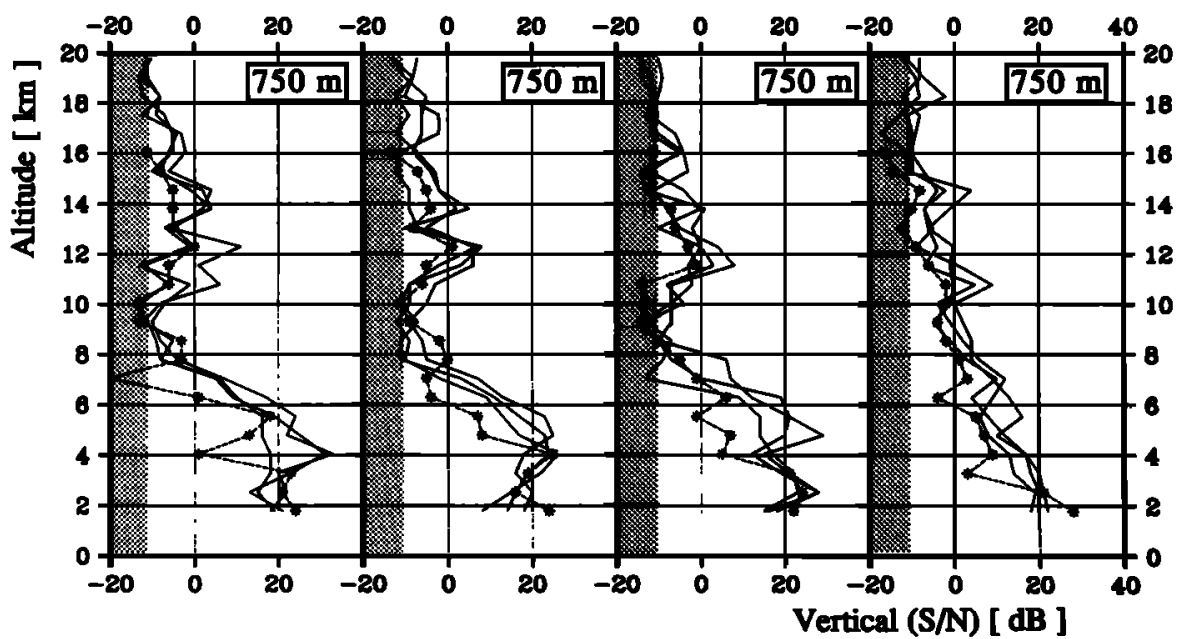

Fig. 7. Vertical profiles of measured (solid line) and reconstructed (dashed line with asterisks) signal to noise ratio (S/N) for vertical radar measurements. The regions where the radar echoes cannot be distinguished from the noise are shaded. The reconstruction process is here based on rawinsonde measurements of the vertical gradient of potential refractive index (taking into account the effect of humidity in the troposphere). The range resolution is always $\Delta r=750 \mathrm{~m}$ and the three radar profiles in each panel are separated by half an hour (this allows estimation of the time variability for vertical measurements). The comparison gives similar results as in Figure 6 except for some discrepancies in the troposphere, probably linked with poor humidity measurements (saturation of the sensor). 
to a stronger echo (see the appendix, section A.5). VanZandt and Vincent [1983] suggested that the vertical echoes could be due to the spectrum of gravity waves. This interpretation is rather different than the proposed one (see the discussion in section 3.5), but in both cases, the $\mathrm{N}_{\mathrm{BV}}^{4}$ dependency results from adiabatic vertical displacements. In their model, a slight increase (0.5) of the exponent is a consequence of the variation of the total energy with the local stability assumed in the model of VanZandt [1982]. While the anisotropy of their model spectrum does depend on the local stability, as assumed here, this effect has no consequences on their exponent, since the radar lobe was assumed to be "large" with respect to the model spectrum.

However, the nearly perfect level agreement in Figure 6 and 7 must be considered as spurious because of the spectrum anisotropy. The effect of this anisotropy, as estimated in section 3.6, is to increase by an unknown, but large (order of $10^{2}$ ) factor, the predicted level. At the present time, we have no satisfactory explanation for this huge discrepancy.

\subsection{The poor oblique reconstruction}

The problem of the shape agreement, i.e., the altitude and relative importance of maxima and minima, without consideration of level or dynamic range, can be understood, in the same way as for vertical reconstruction, as an effect of horizontal and temporal extent of vertical structures. Furthermore, the turbulent temperature fluctuations used here have probably a smaller extent than the mean temperature gradients used for the vertical comparison, because of the intermittence of the turbulent patches.

In order to increase the small dynamic range of the reproduced oblique signal, it is possible to suppose, as already suggested for the vertical comparison, that the turbulent inertial three-dimensional spectrum is not isotropic for scales $\approx 3 \mathrm{~m}$. This assumed anisotropy would have to depend on local properties of the medium (stability, wind shear, etc.) and should be far smaller than the vertical one (otherwise it would have been detected by the radars). This hypothesis, while not unreasonable, is largely speculative and in any case is quite "ad hoc." Furthermore, as for the vertical case, the introduction of a moderate anisotropy in the spectrum would increase the level discrepancy (see Figure A1 in the appendix).

It is possible that noise contamination from the temperature sensor (see Figure 3) could mask the true spectrum at the observed scale. This can occur for weak temperature fluctuations and would produce both an overestimation of the minima level in the reconstructed profiles and a reduction of the dynamic range. However, a careful examination of the individual spectra shows that this circumstance is quite exceptional. An attempt was made to subtract this noise level from the spectrum before correction for the time constant, but no improvement resulted in the reconstructed profiles.

\subsection{Uncertainties}

We will start this discussion with an examination of the possible error sources in order to establish clearly that the observed discrepancy is real.

For in situ measurements, some error sources can be listed. The altitude and vertical velocity of the balloon are deduced by two independent methods (radar tracking and integration of the hydrostatic equation); the results are consistent and can be considered as accurate. Temperature measurements are obtained from two micro bead thermistors, carefully calibrated before the flight. Their indications coincide with those of the coarse temperature sensors and with nearby radio soundings; thus only high-frequency perturbations can be suspected. An attenuation is indeed observed as a result of the time constant of the sensor. The temperature spectra are corrected for this attenuation and an error in the estimation of the time constant could produce a spectral level and slope variation. The time constant is calculated as a function of density from the physical characteristics of the sensor and this theoretical determination was checked during the flight when the sensor occasionally slipped into the shadow of the gondola, and was thus subjected to a step of temperature. Another check is possible in some turbulent layers where the temperature spectrum retains the same $-5 / 3$ slope (after correction) throughout the attenuated range down to the noise level. As discussed earlier, this noise level can lead to an overestimation of the spectral level in regions where the latter is very low; however, these situations are quite exceptional. Furthermore, the observed dynamic range of the $\mathrm{C}_{\mathrm{n}}^{2}$ parameter (Figure 4), deduced directly from the spectral level at $k=2 \mathrm{rad} \mathrm{m}^{-1}$, is larger than one decade at any altitude. This constitutes strong evidence that the spectrum is well over the noise level for this scale range.

The comparison between in situ and radar measurements is made through (1) and (5), the latier relying on the isotropy hypothesis. The very existence of an aspect sensitivity for radar measurements shows that this hypothesis is certainly wrong for the vertical comparison, while for the oblique one it appears quite reasonable. The presence of this known anisotropy constitutes the only cause of (serious) discrepancy, for vertical measurements, between the theoretical predictions, based on measured temperature (and humidity) gradients, and the observed $\mathbf{S} / \mathbf{N}$ profile. Equation (1) contains some factors that cannot be precisely determined. The radiated power $\alpha P_{t}$ is known with an uncertainty of $\approx 50 \%$ because the various resistive losses and the power attenuation $\alpha$ are not well known. The effective area of the antenna $A_{e}=4 / 9 A_{g}$ is deduced from a theoretical calculation and is not precisely known. The associated error can be as large as $30 \%$.

The systematic error, linked with the use of a constant noise temperature $T_{N}=6700 \mathrm{~K}$ instead of an interpolated one, is small on Figure $7(-0.7$ to $+0.3 \mathrm{~dB})$ but corresponds to a shift of the calculated profile of $+1.6 \mathrm{~dB}$ on Figure 6 and $+2.6 \mathrm{~dB}$ on Figure 5. This effect tends to increase the $S / N$ discrepancy for oblique measurements. The statistical error associated with the interpolation between the three temperature maps is $\approx 10 \%$.

The maximum integration time used in this experiment $\tau=0.624 \mathrm{~s}$ can be compared to the observed decorrelation time of the medium, larger than $1 \mathrm{~s}$ (as deduced from the Doppler width of the echoes). The corresponding error on the associated number of coherent integration $\mathbf{N}_{C I}$ is thus small. The other coefficients in equation (1) are well known and cannot contribute significantly to the discrepancy.

Thus, the global uncertainty in the comparison between reconstructed and measured profiles of $S / N$ is smaller than a 
factor 2 or 3 (less than $5 \mathrm{~dB}$ ), and the observed discrepancy must be considered as real.

\subsection{Discussion}

These discrepancies are in the same direction for the oblique and the vertical comparison (an overestimation of the reconstructed profiles), taking into account the known (though not quantitatively) effect of the spectrum anisotropy. It is difficult to say whether they are of the same order for the two directions; a crude estimate of the effect of anisotropy suggests a positive answer. It is worth noting that if the losses in the radiating elements of colinear-coaxial antennas (included in $\alpha$ ) are underestimated, the discrepancy observed in both directions would be reduced.

To our knowledge, the present work is the first attempt to make a direct comparison, without any adjustable parameter, between an ST radar and nearby in situ measurements. Thus it is difficult to compare it to other published results.

For oblique measurements, VanZandt et al. [1978] proposed a statistical model in order to reconstruct $C_{n}^{2}$, and thus $\mathbf{S} / \mathbf{N}$, profiles. This model uses the classical hypothesis of inertial and isotropic turbulence, and relates the "external scale" $L_{0}$ to the local $\mathrm{C}_{\mathrm{n}}^{2}$ parameter. It then computes the probability of occurrence of a turbulent layer as a function of the mean properties of the medium, using a Richardson stability criterion. This model uses rawinsonde data and predicts a profile of mean $\mathrm{C}_{\mathrm{n}}^{2}$ whose agreement with the radar measurements is quite good. The parameter $L_{0}$ is used as an adjustable constant; the value leading to the best fit with experimental data $(10 \mathrm{~m})$ appears very comparable with direct in situ measurements [Barat and Bertin, 1984] or with stellar scintillation data [Coulman et al., 1988]. However, the hypothesis that no temperature fluctuations exist in regions where $\mathrm{Ri}>1 / 4$ seems very unrealistic. Such fluctuations correspond to decaying or "fossil" turbulence and their inclusion in the model would have led to an overestimation of the predicted $\mathrm{C}_{\mathrm{n}}^{2}$.

As regards vertical measurements, the Fresnel scattering model of Gage et al. [1985] produces an equivalent reconstruction of the observed radar reflectivity profiles. This model assumes, as in the present work, that the refractive index fluctuations result from vertical displacements in a stably stratified medium, and thus implicitly predicts the same dependency of radar reflectivity upon the local static stability $\left(\mathrm{N}_{\mathrm{BV}}^{4}\right)$. However, this comparison uses a "Fresnel reflection" formula, equivalent to an "infinite anisotropy" hypothesis [Doviak and Zrnic', 1984], and adjusts the observed and predicted profiles with an empirical coefficient.

\subsection{Conclusions}

When the radar equation is directly used with in situ measurements in order to reconstruct the observed $S / N$ profile, some surprising results are found:

For oblique measurements, where the classical isotropy hypothesis is supposed to be valid, the reconstruction is very disappointing. The shape is poorly reproduced, the dynamic range is smaller than observed, and the $\mathbf{S} / \mathrm{N}$ is strongly overestimated. For this last point, we could not find any satisfactory explanation. For the first two points, the highly intermittent nature of turbulence allows us to understand why statistical models, based on mean properties of the medium, with presumably large horizontal extension, give satisfactory results when compared with medium vertical resolution, time averaged, radar measurements. It is clear that the dynamic range observed on both in situ and radar high-resolution profiles results partly from an important time variability, and that a time average would reduce it.

As regards vertical measurements, the hypothesis used, namely the predominance, at scales $=3 \mathrm{~m}$, of an anisotropic temperature spectrum due to the vertical displacement of fluid particles in a stably stratified background, appears very satisfactory regarding the reconstruction of the shape and dynamic range observed with the radar. However, the predicted level is strongly overestimated, as for oblique measurements, when the anisotropy is included, even qualitatively, in the reconstruction process. This constitutes a puzzling maze, since the buoyancy range theory is in perfect agreement with in situ measurements [Sidi and Dalaudier, 1988] and since the aspect sensitivity of the radar echoes constitutes a proof of this anisotropy and gives some clues to its order of magnitude.

The general conclusions of this work cannot be considered as definitive, since they are based on a single comparison which was made with a small number of radar profiles (with no accurate calibration) and only one balloon ascent (and some rawinsondes profiles). However, to our knowledge, this work is the first attempt to make a quantitative assessment of the radar equation (1). The partial failure of this comparison, especially for the determination of the level of signal returns, can be considered as a warning for a cautious use of the radar equation.

This failure of a direct comparison between individual measurements does not contradict the success of such reconstruction for the mean radar profile based on large-scales properties of the medium. However, up to now, such models used arbitrary constants in order to adjust the predicted level to the observed one, and thus, do not constitute an assessment of the coefficients of equation (1).

From a pragmatic point of view, the success of the reconstruction obtained in Figure 6 and 7, when ignoring the effect of the anisotropy, can be viewed as a new empirical model for the vertical VHF radar measurements. This model, while based on a different hypothesis, is similar to the one proposed by Gage et al. [1985], except that no adjustment constants are needed.

A more detailed comparison between in situ and remote sensing measurements of the temperature (or refractive index) fluctuations is currently planned in April 1989 with the participation of other instruments.

\section{APPENDIX}

\section{A.1. Definitions}

In this appendix, the relations between three-dimensional, one-dimensional and scalar spectra are established for the case of a simple anisotropic model. The first assumption is that the anisotropic auto correlation function (ACF) $R_{a}$ corresponds to 
an isotropic one $\mathbf{R}_{\mathbf{i}}$ flattened in the vertical direction. In other words the "iso-ACF" are oblate ellipsoids with an aspect ratio $\beta$ independent of $k$. This can be written

$$
\mathbf{R}_{\mathrm{a}}(\mathrm{x}, \mathrm{y}, \mathrm{z})=\mathbf{R}_{\mathrm{i}}(\mathrm{x}, \mathrm{y}, \boldsymbol{\beta} \mathrm{z})
$$

The second assumption is that the one-dimensional vertical spectrum $S_{i}$ associated with $R_{i}$ is a power law with a spectral index $p$. If $\phi_{i}$ is the associated isotropic three-dimensional spectrum, this means

$$
S_{i}=A k^{p} \quad \phi_{i}=-\frac{p A}{4 \pi} k^{(p-2)}
$$

The anisotropic three-dimensional spectrum $\phi_{\mathrm{a}}$ is the Fourier transform of $R_{\mathrm{a}}$. Thus, with $\mathrm{z}^{\prime}=\boldsymbol{\beta} \mathrm{z}$

$$
\begin{aligned}
& \begin{aligned}
\phi_{a} & =\iiint R_{a}(x, y, z) e^{i} k r d x d y d z \\
\quad & \iiint R_{R_{i}}\left(x, y, z^{\prime}\right) e^{i\left(k_{x} x+k_{y} y+k_{z} z^{\prime} / \beta\right)} d x d y d z / \beta
\end{aligned} \\
& \phi_{a}\left(k_{x}, k_{y}, k_{z}\right)=(1 / \beta) \phi_{i}\left(k_{x}, k_{y}, k_{z} / \beta\right)
\end{aligned}
$$

\section{A.2. The one-dimensional vertical spectrum}

By definition, the one-dimensional spectrum is

$$
\begin{aligned}
S_{\mathrm{a}}\left(k_{\mathrm{z}}\right) & =2 \iint \phi_{\mathrm{a}} d \mathrm{k}_{\mathrm{x}} d \mathrm{k}_{\mathrm{y}} \\
& =-\frac{\mathrm{p} A}{2 \pi \beta} \iint\left(\mathrm{k}_{\mathrm{x}}^{2}+\mathrm{k}_{\mathrm{y}}^{2}+\mathrm{k}_{\mathrm{z}}^{2} / \beta^{2}\right)^{(\mathrm{p}-2) / 2} d \mathrm{k}_{\mathrm{x}} \mathrm{dk_{y }}
\end{aligned}
$$

with the variable change $k_{x}^{2}+k_{y}^{2}=r^{2}, d k_{x} d k_{y}=d\left(\pi r^{2}\right)$ and $k_{2}^{2} / \beta^{2}=c^{2}$ the two fold integral is

$$
\pi \int_{0}^{\infty}\left(r^{2}+c^{2}\right)^{(p-2) / 2} d\left(r^{2}\right)=\pi \int_{c^{2}}^{\infty} t^{(p-2) / 2} d t=-\frac{2 \pi}{p} c^{p}
$$

and thus

$$
S_{\mathrm{a}}\left(\mathrm{k}_{\mathrm{z}}\right)=(\mathrm{A} / \beta)\left(\mathrm{k}_{\mathrm{z}} / \beta\right)^{\mathrm{P}}
$$

\section{A.3. The scalar spectrum}

In spherical coordinates $(k, \theta, \varphi)$, the scalar spectrum reads

$$
\begin{gathered}
E_{a}(k)=-\frac{p A}{4 \pi \beta} \iint \Re k^{2} \sin \theta d \theta d \varphi \\
R=\left(k^{2} \sin ^{2} \theta \sin ^{2} \varphi+k^{2} \sin ^{2} \theta \cos ^{2} \varphi+k^{2} \cos ^{2} \theta / \beta^{2}\right)^{(p-2) / 2} \\
E_{a}(k)=-\frac{p A}{\beta} k_{0}^{p} \int_{0}^{1}\left[1+\left(\cos ^{2} \theta\right)\left(1 / \beta^{2}-1\right)\right]^{(p-2) / 2} d(\cos \theta) \quad(A 5)
\end{gathered}
$$

This shows that the scalar spectrum is a power law as in the isotropic case. Setting $m=\left(1 / \beta^{2}-1\right)$ and $\chi=\cos \theta$, the integral in the right-hand side reads

$$
F_{p}(\xi)=\int_{0}^{\xi}\left[1+m \chi^{2}\right]^{(p-2) / 2} d \chi
$$

For the special case $p=-3$, this function is known to be

$$
F_{-3}(\xi)=\left(\frac{\xi^{2}}{1+m \xi^{2}}\right)^{1 / 2}-\frac{m}{3}\left(\frac{\xi^{2}}{1+m \xi^{2}}\right)^{3 / 2}
$$

and the scalar spectrum reads

$$
E_{a}(k)=\frac{3 A}{\beta} F_{-3}(1) k^{-3}=\left(2+\beta^{2}\right) A k^{-3}
$$

\section{A.4. Relation for strong anisotropy}

For a given $k$, the ratio between the vertical and the horizontal value of the three-dimensional spectrum is $\beta^{(2-p)}$. For values of $p$ smaller than -1.5 and values of $\beta$ larger than 5 [Waterman et al., 1985] this ratio is larger than $\approx 300$. With such a strong anisotropy, the only region contributing to the integral for both one-dimensional and scalar spectra is the region close to the vertical axis. In this region, the two integration surfaces differ very slightly (a sphere and its two tangent planes are very close near the axis of symmetry). Thus, for large anisotropy, the vertical one-dimensional spectrum and the scalar spectrum are (nearly) identical.

This relation can be verified in the case $p=-3$, where exact integration was performed, as $\beta^{2}$ is very close to $\left(\beta^{2}+2\right)$ for large values of $\beta$.

\section{A.5. Variation of the radar signal with the anisotropy}

As stated in section 3.1, the radar signal, as a function of beam pointing angle, is proportional to the angular convolution of the three-dimensional spectrum with the radar lobe. In order to simplify the computation, an equivalent "conic lobe" (with an efficiency of 1 inside the lobe and 0 outside) of total width $5.2^{\circ}$ is used. The convolution then reduces to a simple integration of the three-dimensional spectrum into the solid angle associated with the lobe. In order to study the variation of the signal with the anisotropy, for a given scalar spectrum $E$, this integral must be normalized by two factors. First, it must be divided by the solid angle corresponding to the integration domain, thus giving a quantity equivalent to a three-dimensional spectrum. Then, in order to obtain a dimensionless quantity, it must be divided by the isotropic three-dimensional spectrum that would produce the assumed scalar spectrum E. Using (A6), this normalized signal reads

$$
G_{p}(\beta)=\frac{F_{p}\left(c_{1}\right)-F_{p}\left(c_{2}\right)}{\left(c_{1}-c_{2}\right)} \frac{(1-0)}{F_{p}(1)-F_{p}(0)}
$$

where $c_{1}$ and $c_{2}$ are the cosines of the integration limits $\left(0^{\circ}\right.$ and $2.6^{\circ}$ for vertical measurements or $11.9^{\circ}$ and $17.1^{\circ}$ for oblique ones). Using this simple model, an estimate of the radar signal can be obtained in two steps: First, starting with either an in situ measurement or a theoretical estimate of the temperature spectrum, the echo power is computed using (1) along with the isotropic conversion formulas (5). Second, the 


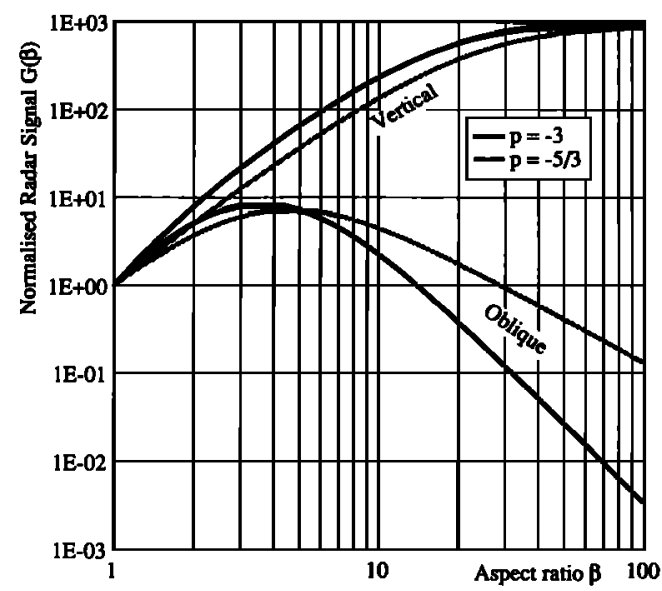

Fig. A1. Variation of the expected radar signal as a function of the assumed anisotropy of the temperature three-dimensional spectrum. The calculation is made with the simple anisotropic model stated in the appendix, for vertical and oblique measurements and for the two spectral slopes $p=-3$ (solid) and $p=-5 / 3$ (shaded). The finite size of the radar lobe is taken into account and leads to a saturation effect for vertical measurements, when the "width" of the spectrum is smaller than the lobe. For oblique measurements, after an initial increase for moderate anisotropy, a rapid decrease of the signal is expected for large anisotropies, when all the energy of the spectrum concentrates around the vertical direction.

correction factor $G_{p}(\beta)$ is applied in order to account for the assumed anisotropy of the scattering medium.

For any given spectral slope $p$, this function can be computed by numerical integration. Its variations are shown on Figure A1 (in log-log coordinates) for both vertical and oblique direction and for two values of the spectral slope: $p=-3$ and $p=-5 / 3$. It appears that, for vertical measurements, the finite size of the radar lobe limits the effect of the anisotropy. For values of $\beta$ larger than $\approx 30$, both signals are close to the saturation value, $1 /\left(1-\cos \left(2.6^{\circ}\right)\right),=970$ times the isotropic level. For $p=-3$ and $\beta \approx 5$, the increase of the vertical signal is $\approx 65$; this is only $10 \%$ of the increase of the corresponding three-dimensional spectrum in the vertical direction $\left(\beta^{4}=625\right)$.

For oblique measurements, the signal increases up to a maximum for both spectral slopes $\left(G_{-3}(3.6) \approx 8.3, G_{-5 / 3}(4.6)\right.$ - 7.2) and then decreases rapidly (with a slope $p$ for large $\beta$ ). Since the presumably isotropic scalar spectrum, as deduced from in situ measured turbulence (with a $-5 / 3$ slope), is usually more than a decade above the theoretical BSR spectrum (with a -3 slope) as given by (6), the effect of the anisotropy cannot be seen in the oblique direction (with $k \approx 2 \mathrm{rad} \mathrm{m}^{-1}$ ), even for large values of $\beta$.

Acknowledgments. We wish here to thank the Centre National de la Recherche Météorologique (CNRM) for the meteorological data from the " $4 \mathrm{M}^{\prime \prime}$ station of Duhort. Operation of the balloon and data acquisition was performed by the Centre National d'Etudes Spatiales (CNES), the instrumented gondola was prepared by J. C. Génie. This work was made possible by the financial support of Institut National des Sciences de l'Univers (INSU) (operation of radar "Provence"), Direction des Recherches et des Etudes Techniques (DRET), and Actions Thématiques Programmées (ATP) "Front 84" and "Atmosphère moyenne".

\section{REFERENCES}

Azouit, M., J. Vemin, R. Barletti, G. Ceppatelli, A. Righini, and $N$. Speroni, Remote sensing of atmospheric turbulence by means of a fast optical method: A comparison with simultaneous in situ measurements, J. Appl. Meteorol., 19. 834-838, 1980.

Balsley, B. B., and V. L. Peterson, Doppler-radar measurements of clear air atmospheric turbulence at 1290 MHz, J. Appl. Meteorol., 20, 266-274, 1981.

Barat, J., A high resolution ionic anemometer for boundarylayer measurements, J. Appl. Meteorol., 21, 1480-1488, 1982.

Barat, J., and F. Bertin, On the contamination of stratospheric turbulence measurements by wind shear, J. Atmos. Sci., 41, 819-827, 1984.

Barat, J., C. Cot, and C. Sidi, On the measurement of turbulence dissipation rate from rising balloons, $J$. Atmos. Oceanic Technol., 1, 270-275, 1984.

Blythe, J. H., Result of a survey of galactic radiation at $38 \mathrm{Mc} / \mathrm{s}$, Mon. Not. R. Astron. Soc., 117, 654-655, 1957.

Briggs, B. H., and R. A. Vincent, Some theoretical considerations on remote probing of weakly scattering irregularities, Aust. J. Phys., 26, 805-814, 1973.

Coulman, C. E., J. Vernin, Y. Coqueugniot, and J. L. Caccia, Outer scale of turbulence appropriate to modeling refractiveindex structure profiles, Appl. Opt., 27, 155-160, 1988.

Dalaudier, F., and C. Sidi, Evidence and interpretation of a spectral gap in the turbulent atmospheric temperature spectra, J. Atmos. Sci., 44, 3121-3126, 1987.

Dewan, E. M., and R. E. Good, Saturation and the "universal" spectrum for vertical profiles of horizontal scalar winds in the atmosphere, J. Geophys. Res., 91, 2742-2748, 1986.

Doviak, R. J., and D. S. Zrnic', Reflection and scatter formula for anisotropically turbulent air, Radio Sci., 19, 325-336, 1984.

Gage, K. S., and B. B. Balsley, On the scattering and reflection mechanisms contributing to clear air radar echoes from the troposphere, stratosphere and mesosphere, Radio Sci., 15, 243-257, 1980.

Gage, K. S., W. L. Ecklund, and B. B. Balsley, A modified Fresnel scattering model for the parametrization of Fresnel returns, Radio Sci., 20, 1493-1501, 1985.

Green, J. L., and K. S. Gage, A reexamination of range resolution dependence of backscattered power observed by VHF radars at vertical incidence, Radio Sci., 20, 1001-1005, 1985.

Green, J. L., K. S. Gage, and T. E. VanZandt, Atmospheric measurements by VHF pulsed Doppler radar, IEEE Trans. Geosci. Electron., 17, 262-280, 1979.

Hey, J. S., S. J. Parsons, and J. W. Philips, An investigation 
of galactic radiation in the radio spectrum, Proc. $R$. Soc. London, Ser. A, 192, 425-445, 1947.

Hocking W. K., Radar studies of small scale structure in the upper middle atmosphere and lower ionosphere, Adv. Space Res., Z(10), 327-338, 1987.

Holloway, G., A conjecture relating oceanic internal waves and small scale processes, Atmos. Ocean, 21, 107-122, 1983.

Landecker, T. L., and R. Wielebinsky, The galactic meter wave radiation, Aust. J. Phys. Astrophys. Suppl., 16, 1-30, 1970.

Lumley, J. L., The spectrum of nearly inertial turbulence in a stably stratified fluid, J. Atmos. Sci., 21, 99-102, 1964.

Monin, A. S., and A. M. Yaglom, Statistical Fluid Mechanics, vol. 2, p. 33, M.I.T. Press, Cambridge, Mass., 1975.

Ottersten, H., Mean vertical gradient of potential refractive index in turbulent mixing and radar detection of CAT, Radio Sci., 4, 1247-1249, 1969a.

Ottersten, H., Radar backscattering from the turbulent clear atmosphere, Radio Sci., 4, 1251-1255, 1969 b.

Rottger, J., Reflection and scattering of VHF radar signals from atmospheric refractivity structure, Radio Sci., 15 , 259-276, 1980.

Röttger, J., J. Klostermeyer, P. Czechowsky, R. Rüster, and G. Schmidt, Remote sensing of the atmosphere, Naturwissenschafien, 65, 285-296, 1978.

Rottger, J., P. Czechowsky, and G. Schmidt, First low-power VHF radar observations of the tropospheric, stratospheric and mesospheric winds and turbulence at the Arecibo Observatory, J. Atmos. Terr. Phys., 43, 789-800, 1981.

Sheen, D. R., C. H. Liu, and J. Róttger, A study of signal statistics of VHF radar echoes from clear air, J. Atmos. Terr. Phys., 47, 675-684, 1985.

Sidi, C., and F. Dalaudier, Temperature and heat flux spectra in the turbulent buoyancy subrange, Pure Appl. Geophys., $130 \times 2 / 3,1989$.

Sidi, C., J. Lefrère, F. Dalaudier, and J. Barat, An improved atmospheric buoyancy wave spectrum model, $J$. Geophys. Res., 93, 774-790, 1988.
Smith, S. P., D. C. Fritts, and T. E. VanZandt, Evidence for a saturated spectrum of atmospheric gravity waves, J. Atmos. Sci., 44, 1404-1410, 1987.

Tatarski, V. I., Wave Propagation in a Turbulent Medium, McGraw-Hill, New York, 1961.

Tsuda, T., T. Sato, K. Hirose, S. Fukao, and S. Kato, MU radar observations of the aspect sensitivity of backscattered VHF echo power in the troposphere and lower stratosphere, Radio Sci., 21, 971-980, 1985.

Tsuda, T., P. T. May, T. Sato, S. Kato, and S. Fukao, Simultaneous observations of reflection echoes and refractive index gradient in the troposphere and lower stratosphere, Radio Sci., 23, 655-665, 1988.

VanZandt, T. E., A universal spectrum of buoyancy waves in the atmosphere, Geophys. Res. Lett., 9, 575-578, 1982.

VanZandt, T. E., and R. A. Vincent, Is VHF Fresnel reflectivity due to low frequency buoyancy waves?, Handbook for MAP, Vol. 9, pp.78-80, Middle Atmosphere Program, University of Illinois, Urbana, 1983.

VanZandt, T. E., J. L. Green, K. S. Gage, and W. L. Clark, Vertical profiles of refractivity turbulence structure constant: Comparison of observations by the Sunset radar with a new theoretical model, Radio Sci., 13, 819-829, 1978.

Waterman, A. T., T. Z. Hu, P. Czechowsky, and J. Röttger, Measurement of anisotropic permittivity structure of upper troposphere with clear air radar, Radio Sci., 20, 1580-1592, 1985.

Weinstock, J., On the theory of temperature spectra in a stably stratified fluid, J. Phys. Oceanogr., 15, 475-477, 1985.

Woodman, R. F., and A. Guillen, Radar observations of wind and turbulence in the stratosphere and mesosphere, J.Atmos. Sci., 31, 493-505, 1974.

M. Crochet, LSEET, Université de Toulon, 83100, Toulon, France.

F. Dalaudier and C. Sidi, Service d'Aéronomie du CNRS, B.P.3, 91371, Verrières le Buisson Cedex, France 